\title{
«Что вы скажете в этой ситуации?» Die soziopragmatische Komponente kommunikativer Kompetenz im Russischunterricht - wie entwickeln, wie bewerten?
}

\section{Wolfgang Stadler (Innsbruck)}

В Аанной статье поднимается вопрос о статусе социопрагматической компетенции на уроках русского языка как иностранного (РКИ), а также ее развитии и оценке с учетом сложности обучения русскому языку в силу его системной специфики. Оконченное онлайн анкетирование преподавателей РКИ в Германии, Австрии, Южном Тироле и Швейцарии показывает, что прагматическим и социолингвистическим особенностям русского языка в школе не уделяют Аостаточного внимания. Поэтому, во-первых, Аля ознакомления буАущих преподавателей РКИ с вышеуказанной темой был проведен ряд семинарских занятий в университете г. Инсбрука, одной из целей которых являлось сознательное развитие социопрагматической компетенции. Во-вторых, учащимся второго курса того же университета был преАложен тест Аополнения речевого акта в соответствии с заданной ситуацией (discourse completion test). Результаты продемонстрировали, что при уровне владения языком В1 немецкоязычные студенты передают информацию менее адекватно и приемлемо, чем билингвы или носители языка. Особенно это заметно по отношению к таким навыкам как «изложение намерения речевого акта», «регистр общения» или «эмоциальность высказывания». Таким образом, автор приходит к выводу, что коммуникативный и деятельностный подход не может и не АОАжен искцючать социопрагматическую компетенцию на уроках русского языка.

Anka Bergmann, Olga Caspers \& Wolfgang Stadler (Hg.)

Didaktik der slawischen Sprachen - Beiträge zum 1. Arbeitskreis in Berlin (12.-14.9.2016)

(C) 2018 innsbruck university press, ISBN 978-3-903187-11-5, DOI 10.15203/3187-11-5 


\section{Soziopragmatik: Begriffsbestimmung}

Soziopragmatische Kompetenz umfasst dem Gemeinsamen europäischen Referenzrahmen für Sprachen (GeR) zufolge neben dem Erwerb von deklarativem (soziokulturellem) und prozeduralem Wissen (dem nötigen Know-how) auch den Erwerb praktischer und interkultureller Fertigkeiten. Konkret sind unter diesen Fertigkeiten soziale, alltägliche, berufliche sowie interkulturelle Fähigkeiten zu verstehen, die es den Lernenden ermöglichen, verbale Handlungen auszuführen. Dabei müssen sie lernen, gewissen sprachlichen, paralinguistischen und nonverbalen kulturspezifischen Regeln und Konventionen zu folgen, also situativ-sprachliche Angemessenheit über die Kulturen hinweg zu berücksichtigen, und verstehen, was effiziente Kommunikation ihnen abverlangt und in einer bestimmten Domäne bedeutet (vgl. GeR 2001: 24-25, 105-106).

Genaugenommen sprechen die Autoren des GeR nicht von einer „soziopragmatischen“, sondern von einer „soziolinguistischen " und einer „pragmatischen “ Kompetenz. Unter ersterer verstehen sie „die soziale Dimension des Sprachgebrauchs“ (ebd.: 118); unter letzterer das Wissen um diskursive und funktionale Kompetenz sowie die Existenz von „trans-/interaktionalen Schemata“ (ebd.: 126). Wie eng die soziolinguistische und pragmatische Kompetenz miteinander verbunden sind, zeigt sich v. a. im Bereich der vom GeR definierten funktionalen Kompetenz, konkret im Bereich der Paarigkeit von initiierenden und respondierenden Gesprächssequenzen bzw. in der Frage, welche „sozialen Routinen" den Gebrauch von (solchen) Redeschritten in der sprachlichen Interaktion bestimmen.

Leech (1983: 11) unterteilt Pragmatik in eine pragmalinguistische und eine soziopragmatische Komponente, wobei er erstere in Bezug zur Grammatik, letztere in Bezug zu den Sozialwissenschaften setzt und diese als „the sociological interface of pragmatics" (ebd.: 10) bezeichnet. Nach Leech hat Pragmalinguistik mit der (Auswahl der linguistischen) Form, den Strategien und den Funktionen sprachlichen Handelns zu tun, während sich Soziopragmatik mit der Performanz und der Interpretation von kommunikativen Handlungen aus der Sicht eines angemessenen sozialen Verhaltens der teilnehmenden KommunikationspartnerInnen beschäftigt. Die enge Beziehung zwischen Pragmatik und Soziolinguistik

Anka Bergmann, Olga Caspers \& Wolfgang Stadler (Hg.)

Didaktik der slawischen Sprachen - Beiträge zum 1. Arbeitskreis in Berlin (12.-14.9.2016)

(C) 2018 innsbruck university press, ISBN 978-3-903187-11-5, DOI 10.15203/3187-11-5 
wird ebenfalls deutlich, wenn wir uns die Definition pragmatischen Wissens nach Bachman \& Palmer (2010: 46-47) vor Augen führen: Neben der funktionalen (illokutiven) Komponente verstehen die Autoren Soziolinguistik als jenen Teil der Pragmatik, der SprecherInnen dabei unterstützt, eigene Äußerungen zu initiieren, zu interpretieren und auf Äußerungen anderer angemessen zu reagieren. Auch Mirzaei, Roohani \& Esmaeili (2012: 82) beziehen sich auf das sprachliche Kompetenzmodell von Bachman \& Palmer (2010) und verweisen auf Kasper \& Roever (2005), wenn sie sagen: „[S]ociopragmatics encompasses the knowledge of the relationships between communicative action and power, social distance, imposition, and the social conditions and consequences of what you do, when, and to whom."

Wenn ich im Folgenden den Begriff „Soziopragmatik“ verwende, so verstehe ich darunter aufgrund der engen Relation zwischen Pragma- und Soziolinguistik immer beide Komponenten - sowohl die pragmalinguistische als auch die soziolinguistische. Lernende verfügen über soziopragmatische Kompetenz aus ihrer L1 (vgl. z. B. Marasco, O’Rourke et al. 2004'); es gilt, beim Erwerb der Fremdsprache, im vorliegenden Fall des Russischen, ihr Bewusstsein für die vorhandene Kompetenz zu wecken, sie in der Fremdsprache weiterzuentwickeln und v. a. in Hinblick darauf zu schärfen, welche Fähigkeiten, Fertigkeiten und Strategien sie aus der Erstsprache (L1) auf die L2 (L3, L4 ...) übertragen können (vgl. Kasper \& Rose 2001: 4). Es macht vermutlich wenig Sinn, im Russischunterricht zwischen Pragmatik und Soziolinguistik zu trennen, gilt Russisch doch unter vielen Lernenden - und selbstverständlich auch unter den Lehrenden - als schwierige Sprache, die bei ihrer Vermittlung und Aneignung allein im Bereich der Schrift, der Phonetik, Morphologie, Syntax und Lexik genug Herausforderungen an Lehrkraft und SchülerInnen stellt (vgl. Madyarov 2013: 2058). Bleibt da Zeit, auf soziopragmatische Besonderheiten einzugehen, diese zu vermitteln und auch zu überprüfen?

1 Marasco, O'Rourke et al. (2004: 3-4) nennen neben communicative intentions und engagement noch zwei weitere skills, nämlich nonverbal rules und verbal rules of conversation, als die im Rahmen der L1-Pragmatik zu beurteilenden sprachlichen Fertigkeiten bei Kindern im Vorschulalter. Werden diese Fertigkeiten nicht oder nur teilweise in der L1 erworben, zeigen sich auch in anderen Sprachen pragmatische Schwächen oder Fehlleistungen.

Anka Bergmann, Olga Caspers \& Wolfgang Stadler (Hg.)

Didaktik der slawischen Sprachen - Beiträge zum 1. Arbeitskreis in Berlin (12.-14.9.2016)

(C) 2018 innsbruck university press, ISBN 978-3-903187-11-5, DOI 10.15203/3187-11-5 
In einer Online-Fragebogenerhebung zur Leistungsbeurteilungskompetenz von RussischlehrerInnen in Deutschland, Österreich, Südtirol und der Schweiz (Drackert \& Stadler 2017) geben 63\% der befragten Lehrkräfte an, dass sie - auf einer Skala von 1 („nie“) bis 4 („oft“) - soziopragmatische Kompetenz entweder „selten“ oder „nie“ im Schulhalbjahr überprüfen, während z. B. Vokabeln nur von 9\% der LehrerInnen „selten“ oder „nie“ abgefragt werden. Russische Grammatik hingegen wird von 95\% der Lehrkräfte „manchmal bzw. oft“ im Halbjahr bewertet, lediglich knapp 5\% beurteilen Grammatikkenntnisse „selten oder nie“. ${ }^{2}$

\section{Ein kurzer Forschungsüberblick}

Es gibt nur wenige Arbeiten zur Entwicklung soziopragmatischer Kompetenz und ihrer Bewertung im Russischunterricht, die einen methodisch-didaktischen Hintergrund haben. So legen z. B. Kolotova \& Kofanova (2012) und Stadler (2015a, 2015b) Untersuchungen zur Entwicklung pragmatischer Kompetenz bzw. zum Testen soziopragmatischer Kompetenz im Russischunterricht vor. ${ }^{3}$ Kolotova \& Kofanova (2012: 3) sehen die kommunikative und pragmatische Kompetenz untrennbar miteinander verbunden. Vernachlässigt man die pragmatische Komponente im Russischunterricht, so führe dies ihrer Meinung nach dazu, dass die Lernenden die sprachlichen Strukturen weder aufgabenspezifisch noch situationsadäquat einsetzen können. Die korrekte Auswahl sprachlicher Mittel, um in der Kommunikation so effizient wie möglich bestehen zu können, müsse bereits im Anfangsunterricht geübt werden und damit Ziel eines jeden Fremdsprachenunterrichts sein. Am Beispiel von zwei Dialogen illustrieren die

2 Die Antworten sagen allerdings nicht aus, warum auf das Überprüfen von soziopragmatischer Kompetenz verzichtet wird (z. B. aus zeitlichen Gründen, aus mangelndem Bewusstsein oder der vermeintlich geringen Relevanz für den Unterricht).

$3 \mathrm{Zu}$ anderen Arbeiten, wie soziopragmatische Besonderheiten im Russischunterricht und zum Zweck der Bewertung eingesetzt werden können, s. z. B. Stadler 2015b. Ein Beitrag von Milejko \& Stacenko (2015) mit dem Titel Formirovanie sociokul'turnoj kompetencii na zanjatijach po russkomu jazyku kak inostrannomu widmet sich eher der Überprüfung landeskundlicher Kenntnisse als der Bewertung soziopragmatischen Wissens.

Anka Bergmann, Olga Caspers \& Wolfgang Stadler (Hg.)

Didaktik der slawischen Sprachen - Beiträge zum 1. Arbeitskreis in Berlin (12.-14.9.2016)

(C) 2018 innsbruck university press, ISBN 978-3-903187-11-5, DOI 10.15203/3187-11-5 
Autorinnen, wie SprecherInnenintention, AdressatInnenreaktion und die Wahl der sprachlichen Mittel, eine Bitte zu formulieren, in einem Alltagsgespräch mit einem/einer FreundIn bzw. einem/einer Fremden umgesetzt werden können, um Lernende für (sozio-)pragmatische Besonderheiten zu sensibilisieren (Kolotova \& Kofanova 2012: 4-5). Die Aufgaben zu den Dialogen sind sprechaktbezogen; die Lernenden sollen die Gemeinsamkeiten und Unterschiede in den verschiedenen sprachlichen Realisierungen direktiver Äußerungen aufzeigen und auf einer Likert-Skala von 1-5 die von ihnen verstandene Einstellung zur Beziehung der GesprächspartnerInnen untereinander, das Ausmaß der Bitte und den Grad der Höflichkeit markieren.

Stadler (2015a, 2015b) diskutiert die Frage unterschiedlicher Formen von Authentizität, die sich im Russischunterricht hinsichtlich Materialien, Aufgaben und Situation/Interaktion stellen. Die Sensibilisierung für soziopragmatische Besonderheiten erfolgt in seinen Beiträgen zunächst über den Zugang der Textauthentizität, die durch Interjektionen und Graduierungs- bzw. Abtönungspartikel erhöht wird, weil dadurch die Beziehungsebene der SprecherInnen besser zum Ausdruck kommt (ebd. 2015a). Während diese lexikalischen Mittel, die einen Einfluss auf die Intonation und Satzmelodie der SprecherInnen haben, von NichtmuttersprachlerInnen (NMS) sehr wohl erkannt und in ähnlichen Situationen auch reproduziert werden können, zeigt sich bei der Überprüfung der Produktion von entsprechenden Sprechakten, dass NMS auf diese sprachlichen Mittel selten zurückgreifen. Die Authentizität des sprachlichen Ausdrucks von NMS weicht deutlich von der voice authenticity der MuttersprachlerInnen (MS) ab. Eine von Stadler (2015b) vorgeschlagene Auswahl an Testitems, die auf dem Format eines Written Discourse Completion Tests (WDCT) basieren, sind größtenteils lexik- und sprechaktbasiert und begünstigen, wie die Ergebnisse zeigen, MS und HerkunftssprecherInnen (HS) - eine Tatsache, die nicht beabsichtigt war, jedoch klar in der Auswertung der Ergebnisse auch der vorliegenden Untersuchung zutage getreten ist (s. Abschnitt 5.3 und 5.4). Lernende des Russischen haben große Schwierigkeiten bei der Lösung solcher WDCT-Items, wenn lexikalisches, illokutives und soziolinguistisches Wissen gleichermaßen anzuwenden sind: „Očevidno, čto sovmestit’ leksičeskij material, illokutivnuju kompetenciju i jazykovuju vežlivost' v odnom testovom zadanii, ne upustiv pri ètom iz polja

Anka Bergmann, Olga Caspers \& Wolfgang Stadler (Hg.)

Didaktik der slawischen Sprachen - Beiträge zum 1. Arbeitskreis in Berlin (12.-14.9.2016)

(C) 2018 innsbruck university press, ISBN 978-3-903187-11-5, DOI 10.15203/3187-11-5 
zrenija namerenie sobesednika i sochranjaja tvorčeskij podchod k rešeniju zadači, sostavilo trudnost' dlja učaščichsja." (Stadler 2015b: 368).

Neben den spezifisch auf das Russische ausgerichteten methodisch-didaktischen Untersuchungen von Kolotova \& Kofanova (2012) und Stadler (2015a, 2015b) sei hier noch überblicksartig eine Reihe von Arbeiten vorgestellt, die sich der soziolinguistischen Kompetenz (Krulatz 2014, 2015 - ebenfalls für das Russische), der pragmatischen (Kanik 2013, Xiao 2015) und soziopragmatischen Kompetenz (Blattner \& Fiori 2011) in anderen Sprachen widmen. So untersucht Krulatz (2014) im Rahmen eines Discourse Completion Tests (DCT) 164 E-MailAnfragen von Studierenden an ProfessorInnen und deren Unterschiede in der strategischen Realisierung durch MS und NMS und kommt zu dem Schluss, dass auch sehr fortgeschrittene LernerInnen Defizite im Bereich der soziolinguistischen Kompetenz erkennen lassen. In einer Folgeuntersuchung (Krulatz 2015) wird die Wirkung dieser E-Mail-Anfragen auf russische MS hinsichtlich Verständlichkeit, Angemessenheit und Höflichkeit untersucht. Krulatz (2015) folgert aus ihrer Analyse, dass sowohl hinsichtlich Verständlichkeit, Angemessenheit und Höflichkeit MS signifikant höhere Werte als NMS aufweisen. Die größten Unterschiede in der sprachlichen Realisierung von Anfragen (requests) zwischen MS und NMS zeigen sich in den Formen der Anrede, dem Briefende, der Begründung für die Anfrage, der Verwendung negierter Formen und des Konditionals sowie der Groß-/Kleinschreibung des Personalpronomens Vy (Sie).

Kanik (2013) stellt in seinem Beitrag das häufig eingesetzte Testformat DCT als geeignetes Untersuchungsinstrument in Frage, da seiner Meinung nach damit eher illokutive als soziolinguistische oder interkulturelle Kompetenz überprüft wird. Er (ebd.: 626-627) beruft sich auf Bachman (1990), der in seinem Sprachkompetenzmodell zwischen illokutiver und soziolinguistischer Kompetenz unterscheidet (vgl. auch Bachman \& Palmer 2010) und entwickelt deshalb ein neues Modell, den sog. „umgekehrten DCT“, R-DCT (Reverse Discourse Completion Test), mit dessen Hilfe die von ihm untersuchten ProbandInnen (türkische L2-SprecherInnen) in einer konkreten Situation kontextuelles soziolinguistisches Wissen nachweisen sollen. Den KandidatInnen werden Sprechakte als Stimuli vorgegeben und sie müssen Situationen benennen, in denen diese Sprechakte geäußert werden könnten. Dabei müssen sie auf das Setting, den/die SprecherIn

Anka Bergmann, Olga Caspers \& Wolfgang Stadler (Hg.)

Didaktik der slawischen Sprachen - Beiträge zum 1. Arbeitskreis in Berlin (12.-14.9.2016)

(C) 2018 innsbruck university press, ISBN 978-3-903187-11-5, DOI 10.15203/3187-11-5 
und HörerIn sowie auf den Sprechakt selbst eingehen. Überprüft wird, ob bzw. wie in der beschriebenen Situation der soziale Abstand, die Machtdistanz und der Grad der Gewichtigkeit (Imposition) wiedergegeben werden und wie sich ihr Sprachgebrauchswissen im Vergleich mit muttersprachlichen TürkInnen darstellt.

Blattner \& Fiori (2011:24) leiten ihre Untersuchung mit der Feststellung ein, dass soziopragmatische und multi-literacies-Kompetenzen sowohl im Klassenzimmer als auch in Lehrwerken meist vernachlässigt würden, obwohl sie wesentliche Bestandteile eines modernen Fremdsprachenunterrichts darstellen sollten. Um dem Konzept der multiliteracy in sozialen Netzwerken gerecht zu werden, wird für eine kleine Zahl fortgeschrittener Spanischlernender eine Facebook-Gruppe eingerichtet, die die Aufgabe bekommt, Facebook-Seiten, die mit ausgewählten Lerninhalten der Gruppe korrespondieren, hinsichtlich Begrüßungs- und Verabschiedungsformeln und in Bezug auf Besonderheiten kulturspezifischer Wortschatzverwendung zu analysieren und aus ihrer Lernersicht zu kommentieren. Damit soll sichergestellt werden, dass die Studierenden soziopragmatische Sprachbewusstheit für Spanisch als ihre Zweitsprache entwickeln.

Eine sehr komplexe Forschungsfrage stellt sich abschließend Xiao (2015), wenn er versucht, die Korrelation zwischen sprachlicher Kompetenz (language proficiency) und pragmatischer Kompetenz aufzuzeigen. Zu diesem Zweck untersucht er in einer Metastudie 28 Forschungsarbeiten der Jahre 1992 bis 2013 aus OnlineDatenbanken und kommt zu folgendem Ergebnis: 13 Arbeiten belegen, dass mit steigender Sprachkompetenz auch die pragmatische Kompetenz zunimmt; vier Arbeiten zeigen keine positive Korrelation zwischen sprachlicher und pragmatischer Kompetenz. Der Rest der untersuchten Forschungsarbeiten kommt in Abhängigkeit von den untersuchten pragmatischen Besonderheiten zu divergenten Resultaten. Damit kann Xiao demonstrieren, dass es keine signifikante positive Korrelation zwischen hohem sprachlichen Können von NMS und muttersprachlicher pragmatischer Kompetenz gibt. Die in den Studien angewandten Testverfahren reichen von DCT, Multiple-Choice-Tests (MCT), Cloze-Tests und Inferenztests bis hin zu Rollenspielen und Interviews. Die untersuchten Sprachen sind - neben Englisch - Deutsch, Spanisch, Katalanisch, Japanisch und Thai. Leider gibt es unter den 28 Forschungsarbeiten keine einzige, die Russisch berücksichtigt.

Anka Bergmann, Olga Caspers \& Wolfgang Stadler (Hg.)

Didaktik der slawischen Sprachen - Beiträge zum 1. Arbeitskreis in Berlin (12.-14.9.2016)

(C) 2018 innsbruck university press, ISBN 978-3-903187-11-5, DOI 10.15203/3187-11-5 


\section{Soziolinguistisch-pragmatische Sprachbewusstheit entwickeln}

Wie der kurze Forschungsüberblick in Abschnitt 2 zeigt, wird dem Erwerb und der Überprüfung von soziopragmatischer Kompetenz in der Fachliteratur durchaus Raum gegeben, auch wenn dafür unterschiedliche Begriffe wie „soziokulturell“, „interkulturell“, „soziopragmatisch“, „pragmatisch“ und „soziolinguistisch“ verwendet werden und Russisch als Fremdsprache (RAF) selten oder nicht im Mittelpunkt der Arbeiten steht. Während sich VertreterInnen der Fachdidaktik und der angewandten Linguistik einig sind, dass die soziolinguistisch-pragmatische Komponente in einem modernen, handlungsorientierten und kommunikativ ausgerichteten Fremdsprachenunterricht keinesfalls fehlen darf, belegt die erwähnte Umfrage unter Russischlehrenden in Deutschland, Österreich, der Schweiz und Südtirol (Drackert \& Stadler 2017), dass Soziopragmatik nicht als Hauptziel des Russischunterrichts fungiert - obwohl der Erwerb „soziolinguistischer Kompetenzen“ ausdrücklich Teil des österreichischen Lehrplans für Lebende Fremdsprachen (BMBWK 2004, 2006) ist bzw. „soziokulturelles Orientierungswissen" auch im Bildungsplan Neuere Fremdsprachen der gymnasialen Oberstufe (z. B. für Hamburg) [Freie und Hansestadt Hamburg, Behörde für Schule und Berufsbildung 2009] verankert ist. Nur zwei von 160 Befragten (Drackert \& Stadler 2017) nennen „die Vermittlung von soziokulturellem Wissen“ explizit als Unterrichtsziel; Begriffe wie „Kultur“ und „interkulturell“ werden unter den Unterrichtszielen 50 Mal genannt; „Landeskunde“ 18 Mal. ${ }^{4}$

Auch Krulatz (2015) und Xiao (2015) bestätigen, dass sogar sehr fortgeschrittene LernerInnen in einer Fremdsprache oft Schwierigkeiten haben, sich in einer konkreten Kommunikationssituation angemessen auszudrücken, weil sie die entsprechenden sprachlichen Mittel nicht zur Verfügung haben bzw. die notwendigen Interaktionsmuster (Schemata) nicht kennen. Obwohl Auslandsaufenthalte für Studierende dazu beitragen, im universitären Russischunterricht sozioprag-

4 Nur eine zusätzliche Befragung könnte zeigen, ob bzw. wann unter „Kultur“ bzw. „Kulturkunde“ von den Lehrenden „Landeskunde“ im engen Sinne von „peredača stranovedčeskoj informacii“ (nach Milejko \& Stacenko 2015: 108) verstanden wird.

Anka Bergmann, Olga Caspers \& Wolfgang Stadler (Hg.)

Didaktik der slawischen Sprachen - Beiträge zum 1. Arbeitskreis in Berlin (12.-14.9.2016)

(C) 2018 innsbruck university press, ISBN 978-3-903187-11-5, DOI 10.15203/3187-11-5 
matisches Bewusstsein und auch eine gewisse Kompetenz darin zu entwickeln, zeigt sich gerade im schulischen Russischunterricht, dass das zielsprachliche Umfeld oft fehlt oder dass sich nur erschwert auf adäquaten zielsprachlichen Input zugreifen lässt (vgl. Kasper \& Rose 2001: 4). So werden in der Regel oft eher komplexe sprachliche Strukturen gemeistert als soziopragmatische Kompetenz erworben (vgl. Krulatz 2015: 117).

Der Überblick hat des Weiteren offengelegt, dass in der Fachliteratur pragmalinguistische Kompetenz häufig anhand isolierter Sprechakte (Bitten, Entschuldigungen, Einladungen aussprechen etc.) untersucht wird. Viel seltener werden Routineformeln (kommunikative Phraseme), die diskursive Abfolge von Sprechakten oder die Grenze zwischen propositionalem Gehalt und Implikatur erforscht. Soziolinguistische Kompetenz wird meist mit sprachlicher Höflichkeit gleichgesetzt und der Einsatz unterschiedlicher sprachlicher Register oder sprachlicher Varietäten stellt eher den Ausnahmefall in Analysen zum gesteuerten Spracherwerb dar.

Ich werde mich im nächsten Abschnitt der Frage zuwenden, welche soziolinguistisch-pragmatischen Bereiche den Lernenden im Russischunterricht als Konstrukt nähergebracht werden sollen und beziehe mich dabei primär auf den GeR (2001).

\section{Das soziolinguistisch-pragmatische Konstrukt}

Der GeR spricht von pragmatischer Kompetenz und unterteilt diese in Diskurskompetenz, funktionale Kompetenz und Schemakompetenz; die soziolinguistische Kompetenz umfasst neben Varietäten und unterschiedlichen Sprachregistern auch die sprachliche Kennzeichnung sozialer Beziehungen, Höflichkeitskonventionen und situationstypische Redewendungen (GeR 2001: 119-120, 123, 125-126). Während für die soziolinguistische Kompetenz nur eine Skala („Soziolinguistische Angemessenheit“; ebd.: 121-122) zur Verfügung steht, werden für die pragmatische diskursive Kompetenz vier Skalen angeboten: „Flexibilität“, „Sprecherwechsel“, „Themenentwicklung“, „Kohärenz und Kohäsion“ (ebd.: 124-125). Zwei weitere Skalen sollen die funktionale Kompetenz abdecken: „Flüssigkeit“ und „Genauigkeit“ (ebd.: 129). Unter Schemakompetenz wird 
die Kenntnis sozialer Interaktionsmuster verstanden - ausgehend vom Erwerb simpler paariger Gesprächssequenzen bis hin zu komplexen Skripts, die einzelne Handlungsabfolgen festlegen.

Sowohl bei der Vermittlung als auch bei der Überprüfung soziopragmatischer Kompetenz ist es ratsam, wenn Lehrende den Empfehlungen im Referenzrahmen folgen. Vereinfacht gesagt, bedeutet dies Folgendes: Was ...

- ... kann aus der Erstsprache und anderen Sprachen als bekannt vorausgesetzt werden?

- ... muss von der Lehrkraft ausgewählt und gelehrt werden?

- ... müssen die SchülerInnen selbst erkennen, einschätzen und verstehen?

- ... müssen sie (re)produzieren, beherrschen bzw. selbstständig anwenden können?

Der Inhalt des Konstrukts ist in der folgenden Tab. 1 zusammengefasst und verteilt sich auf alle sechs Kompetenzniveaustufen:

Tabelle 1: Soziolinguistisch-pragmatisches Konstrukt nach GeR

\begin{tabular}{|l|l|}
\hline C1 & Idiomatische Redewendungen \\
\hline B2-C2 & Kohärenz und Kohäsion \\
\hline B2-C1 & Differenziertes Register \\
\hline B1-C2 & Genauigkeit \\
\hline B1-C1 & Flexibilität \\
\hline B1 & Sitten, Gebräuche und Werte \\
\hline A2-C1 & Kennzeichnung sozialer Beziehungen \\
\hline A1-B2 & Situationstypische Redewendungen \\
\hline \multirow{3}{*}{ A1-B1 } & Höflichkeit vs. Grobheit \\
\cline { 2 - 2 } & Neutrales Register \\
\cline { 2 - 2 } & Lineares Verbinden und Verknüpfen von Äußerungen \\
\hline
\end{tabular}

5 Genauer nachzulesen sind die methodisch-didaktischen Überlegungen zum Inhalt des Konstrukts in GeR (2001: 122, 130). 
Wie sich die Progression soziolinguistisch-pragmatischer Inhalte über mehre Stufen hinweg entwickelt, soll im Folgenden anhand der Deskriptoren zu „Sprachliche Kennzeichnung sozialer Beziehungen“ erläutert werden: Während sich auf A1 soziolinguistische Angemessenheit darin zeigt, dass ein elementarer sozialer Kontakt z. B. durch einfachste alltägliche Begrüßungsformeln hergestellt werden kann, so können auf A2.1 soziale Kontakte durch Routineformeln oder auch expressive Sprechakte in der Konversation bewerkstelligt werden. Auf B2 können Beziehungen zu MS aufrechterhalten werden, ohne diese unfreiwillig zu belustigen, zu irritieren oder zu veranlassen, sich anders zu verhalten. AufC1 muss z. B. bei der Fertigkeit des transaktionalen Hörens eine implizit vermittelte Beziehung zwischen GesprächspartnerInnen erkannt bzw. im mündlichen Gespräch Sprache für soziale Zwecke wirksam und flexibel eingesetzt werden.

Es steht außer Zweifel, dass soziolinguistische und pragmatische Fertigkeiten nicht nur im Arbeits-, sondern auch im Alltagsleben eine gewichtige Rolle spielen. ${ }^{6}$ Umso erstaunlicher sind unter diesem Aspekt die Ergebnisse der DESIStudie $^{7}$ im Bereich „Sprachbewusstheit Soziopragmatik“ zu betrachten (vgl. Klieme 2008), welche an SchülerInnen der neunten Jahrgangsstufe aller deutschen Schultypen im Jahr 2003/04 in den Sprachen Englisch und Deutsch durchgeführt wurden. „[K] napp 30\% der Schülerleistungen [verteilen sich] zu Beginn der neunten Jahrgangsstufe auf das Kompetenzniveau A; fast der gleiche Prozentsatz [...] liegt unter diesem Niveau. [...] etwas über $40 \%$ der Schülerleistungen [sind] Niveau B [zuzurechnen], weitere zwei bis drei Prozent liegen auf Niveau C.“ (Nold \& Rossa 2008: 160). Das Testkonstrukt „Sprachbewusstheit Soziopragmatik“ wird für die Sprache Englisch folgendermaßen beschrieben: „Im Mittelpunkt stehen einerseits sprachlich-soziale Fähigkeiten und andererseits Aspekte der Diskurskompetenz, insb. der Sinnkonstruktion in Texten. So soll erkannt und beurteilt werden, ob sprachliche Äußerungen stilistisch ausreichend bestimmten Situationen angepasst sind. Ferner geht es darum, Höflichkeitsanforderungen zu bewerten. Schließlich kommt es darauf an zu erkennen, welche Intentionen Spre-

6 S. z. B. die Studie von Riddiford \& Joe (2010: 195-205), die untersucht, welche Auswirkungen eine Schulung in soziopragmatischen Fertigkeiten für MigrantInnen in Neuseeland bei der Jobsuche hat.

7 Studie zu Unterricht und Kompetenzerwerb in Deutsch und Englisch.

Anka Bergmann, Olga Caspers \& Wolfgang Stadler (Hg.)

Didaktik der slawischen Sprachen - Beiträge zum 1. Arbeitskreis in Berlin (12.-14.9.2016)

(C) 2018 innsbruck university press, ISBN 978-3-903187-11-5, DOI 10.15203/3187-11-5 
cher in ausgewählten Bereichen ausdrücken und wie Sprecherintentionen sich in den Zusammenhang fortlaufender sprachlicher Rede einordnen. Sprachbewusstheit als Kompetenz ist hier demnach darauf ausgerichtet, Facetten sprachlichen Handelns im Diskurs zu erkennen, einzuordnen und zu bewerten. " (Nold \& Rossa 2008: 157).

Die erwähnten Facetten sprachlichen Handelns sind in drei Kompetenzniveaus eingeteilt: A, B und C (s. o.) - wobei es in allen drei Stufen darum geht, sprachliches Handeln zu durchschauen (Hervorhebung W. St.). Differenziert wird zwischen der Vertrautheit des Handlungsablaufs (Kompetenzniveau A), dem Einsetzen von spezifischem fremdsprachlichen Handlungswissen (Kompetenzniveau B) und dem erschließenden Einsetzen von spezifischem fremdsprachlichen Handlungswissen sowie der Bewältigung von Missverständnissen (Kompetenzniveau C). Die in der Studie verwendeten soziopragmatischen Items wurden eigens für diese entwickelt, sie bauen inhaltlich und situativ auf dem relevanten Kontext „Schüleraustausch“ auf, mündliche Rede erscheint in den Testaufgaben verschriftlicht. Argumentiert wird dabei mit dem geringeren Zeitdruck für SchülerInnen (ebd.: 158). Das Testformat, das zum Einsatz kommt, ist ausschließlich MCT. Diese Art der bei der DESI-Studie verwendeten Paper\&PencilTests fanden und finden international auch in der empirischen Forschung Einsatz (vgl. Roever 2014: 129-130), werden aber zunehmend von Computer-Tests und Video-Szenarien abgelöst. Wie aus dem o. a. Konstrukt (Nold \& Rossa 2008) ersichtlich ist, soll neben den sprachlich-sozialen Fähigkeiten auch Diskurskompetenz überprüft werden. Zusätzlich zur sprachlichen Angemessenheit steht „Genauigkeit“ auf dem Prüfstand, allerdings nicht grammatikalische Richtigkeit im Sinne morphologischer und syntaktischer Korrektheit, sondern Genauigkeit des Ausdrucks im Sinne propositionaler Richtigkeit - d. h.: ein/e TestprobandIn bzw. SchülerIn kann auf

- A2-Niveau „bei einem einfachen, direkten Austausch begrenzter Informationen über vertraute Routineangelegenheiten mitteilen, was er/sie sagen will“ (GeR 2001: 129);

Anka Bergmann, Olga Caspers \& Wolfgang Stadler (Hg.)

Didaktik der slawischen Sprachen - Beiträge zum 1. Arbeitskreis in Berlin (12.-14.9.2016)

(C) 2018 innsbruck university press, ISBN 978-3-903187-11-5, DOI 10.15203/3187-11-5 
- B1-Niveau „das Wesentliche von dem, was er/sie sagen möchte, verständlich ausdrücken bzw. die Hauptaspekte eines Gedankens oder eines Problems ausreichend genau erklären" (ebd.) und auf

- B2-Niveau „eine detaillierte Information korrekt weitergeben“ (ebd.).

Die DESI-Studie beschränkt sich auf die Niveaustufen A-B. Niveau C, das - wie gesagt - auch nur zwei bis drei Prozent der untersuchten SchülerInnen aufweisen, würde im Rahmen der propositionalen Genauigkeit die Fähigkeit erfordern, den Grad von Bedeutungsnuancen deutlich zu machen. Auf C1-Niveau beträfe das die Sicherheit/Unsicherheit bzw. den Zweifel/die Vermutung in Bezug auf das Gesagte. Auf C2-Niveau müssten noch feinere Bedeutungsschattierungen hervorgehoben oder Missverständnisse ausgeräumt werden können.

Ich will mich im Folgenden im Bereich der beiden unteren Niveaustufen der selbstständigen Sprachverwendung (B1.1, B1.2) zur Demonstration von möglichen Testitems auf folgende Unterscheidungen konzentrieren: Nähe vs. Distanz, schriftlicher Ausdruck vs. mündlicher Ausdruck, Klassenzimmer- vs. real lifeSituation. Aus einer Reihe von zwölf Items, die im Rahmen von sprachwissenschaftlichen und/oder fachdidaktischen Lehrveranstaltungen an der Universität Innsbruck entwickelt wurden, um Studierende für das Überprüfen soziopragmatischer Kompetenz in ihrem zukünftigen Beruf zu sensibilisieren, werden in der Folge fünf Items vorgestellt, die die Komplexität einer solchen Überprüfung offenlegen. Dabei kommen - anders als in der DESI-Studie - ausschließlich DCItems zum Einsatz. Ziel dieser Darstellung ist es aufzuzeigen, wie vielschichtig sich die Erstellung eines Tests zur Überprüfung soziopragmatischer Kompetenz auf B1-Niveau erweist. Im Mittelpunkt der Items steht mündliche und schriftliche Kommunikation unter Familienmitgliedern, FreundInnen, mit PassantInnen und Angestellten eines Restaurants bzw. Tourismusbetriebes mit dem Zweck zu überprüfen, ob der/die Lernende „das Wesentliche von dem, was er/sie sagen möchte, verständlich ausdrücken“" (ebd.) kann. 


\section{Die Umsetzung des Vorhabens}

Gemeinsam mit Studierenden der Leopold-Franzens-Universität Innsbruck beschäftigte sich der LV-Leiter in drei verschiedenen Lehrveranstaltungen „Schriftlichkeit und Mündlichkeit“ (LV1), „Pragmatik und Soziolinguistik“ (LV2) sowie „Kommunikation - Text - Authentizität“ (LV3) mit linguistischen Themen und ihrer fachdidaktischen Umsetzung. TeilnehmerInnen waren sowohl Lehramtsstudierende des Unterrichtsfaches Russisch als auch Bachelorstudierende aus dem Fachbereich Slawistik. Der Großteil der Studierenden hat Deutsch als Erstsprache, es gibt aber auch MS, deren L1 Russisch ist, und HS, die zweisprachig sind. Während wir uns in LV1 v. a. Fragen der medialen und konzeptuellen Mündlichkeit und Schriftlichkeit von Texten widmeten bzw. dem Problem, wie man Originaltexte für den Schulgebrauch adaptieren oder verschriftlichte Dialogtexte in Lehrwerken ,mündlicher' und somit authentischer gestalten kann, war LV2 insb. von einem awareness raising für soziopragmatische Kompetenz im Russischunterricht (auf A- und B-Niveau) geprägt. Die Lehr-/Lernziele waren vorrangig auf das Konzept der sprachlichen Nähe und Distanz ausgerichtet. Studierende sollten bei der Arbeit mit verschiedenen Lehrwerken und der Simulation von selbst entwickelten Unterrichtssequenzen ihr Bewusstsein für die Vermittlung eben dieser Kompetenz erweitern. LV3 beschäftigte sich primär mit der Erstellung, dem Vergleich und der Verwendung von didaktischen, adaptierten und authentischen Texten im Unterricht und in der Kompetenzüberprüfung. In LV1 wurden Texte gesammelt bzw. erstellt, die auch im schulischen Unterricht zur Demonstration von Unterschieden zwischen schriftlichem und mündlichem Russisch eingesetzt werden können. Manche dieser Texte wurden auch in LV2 und LV3 wiederverwendet; in LV1 und LV2 wurde eine Auswahl dieser Texte als Basis für die Erstellung der Testitems genützt.

\subsection{Die ProbandInnen}

Gruppe 1 setzte sich aus 20 Seminar-TeilnehmerInnen zusammen (18 weiblich, 2 männlich), von denen 13 Deutsch, fünf Russisch, eine Serbisch und eine weitere Studierende Deutsch und Russisch als Erstsprache(n) angaben. Abgesehen von

Anka Bergmann, Olga Caspers \& Wolfgang Stadler (Hg.)

Didaktik der slawischen Sprachen - Beiträge zum 1. Arbeitskreis in Berlin (12.-14.9.2016)

(C) 2018 innsbruck university press, ISBN 978-3-903187-11-5, DOI 10.15203/3187-11-5 
den MS und der HS waren alle TeilnehmerInnen schon mind. drei Jahre oder länger in den gesteuerten Russischunterricht an der Universität eingebunden. Vier Studierende waren jünger als 20 Jahre, zehn waren unter 30, drei unter 40, zwei jünger als 50 und eine über 50 Jahre alt. Zwei Studierende sprachen neben Russisch eine weitere Fremdsprache; elf Studierende sprachen zwei, drei Studierende drei und drei Studierende vier Fremdsprachen. Eine einzige Studierende gab an, neben Russisch noch fünf weitere Fremdsprachen zu sprechen.

Gruppe 2 bestand aus 22 Studierenden eines universitären Sprachbeherrschungskurses (12 weiblich, 10 männlich), die im dritten Semester (zweiten Studienjahr) die LV Russisch II (RII) besuchten. Für je eine/n Studierende/n war Russisch die L1 bzw. L2; für drei Studierende stellte Russisch die L3 dar, für neun Studierende war es die L4, für sechs die L5 und für zwei die L6. Je zehn StudentInnen studierten Russisch erst ein bzw. zwei Jahre; ein/e Studierende/r studierte bereits vier Jahre, ein/e weitere/r war länger als vier Jahre in die Sprachausbildung eingebunden. Drei StudentInnen sprachen neben Russisch eine weitere Fremdsprache; neun Studierende zwei, sechs Studierende vier und vier Studierende vier Fremdsprachen. 20 StudentInnen waren jünger als 30 Jahre; eine/r war unter 40, ein/e weitere/r unter 50 .

\subsection{Erstellung der Testitems}

Das Bestreben, das sich aus den Inhalten und Zielen der LV1-3 entwickelte, war, für verschiedene Kommunikationssituationen in didaktischen, semiauthentischen und authentischen Kontexten Testitems zu erstellen und dabei eine Bewusstheit für soziopragmatische Kompetenz unter zukünftigen LehrerInnen (Gruppe 1) sowohl hinsichtlich Testerstellung als auch -auswertung zu entwickeln. Die für das Prozedere ausgewählten fünf Testitems wurden von Studierenden (BA-Studierende Slawistik und Lehramtsstudierende des Unterrichtsfaches Russisch) z. T. aus folgenden Texten entnommen: Item 1 basiert auf einem didaktischen Text (Kolotova \& Kofanova 2012); Item 2 findet sich in einem Lehrwerktext (Ėsmantova 2011); Item 3 stammt aus einer Textsammlung verschriftlichter Alltagsgespräche (ŽRUG 1995). Item 4 hingegen wurde aus der Erinnerung einer MS rekonstruiert und Item 5 wurde von den Studierenden als eigenes Beispiel

Anka Bergmann, Olga Caspers \& Wolfgang Stadler (Hg.)

Didaktik der slawischen Sprachen - Beiträge zum 1. Arbeitskreis in Berlin (12.-14.9.2016)

(C) 2018 innsbruck university press, ISBN 978-3-903187-11-5, DOI 10.15203/3187-11-5 
für die Testsituation geschaffen. Die Items erfuhren dem Testzweck entsprechend in Folge mehrmals eine Änderung, sie wurden teilweise mit Markern mündlicher Sprache versehen und so für die zu testende Kommunikationssituation adaptiert.

\section{Item 1}

Вы приехали к своему Аругу в Аругой город. На третий Аень вашего пребывания вы возвращаетесь из магазина и обнаруживаете, что вашего Аруга нет Аома, а ключ вы забыли. Вам придётся попросить телефон у общего Аруга из соседней квартиры, с которым вы хорошо знакомы.

Что Вы скажете в этой ситуачии?

Вы:

Abbildung 1: Einen Freund um sein Handy bitten

\section{Item 2}

Прочитайте первую реплику диалога межАу матерью и сыном.

Мать: - Ты уже знаешь, что твой брат сегодня сказац, что он женится!

Как Вы отреагируете в этой ситуачии?

Вы: -

Abbildung 2: Auf eine Neuigkeit in der Familie reagieren

\section{Item 3}

Прочитайте нача^о Аиалога между мужем (A) и женой (Б).

А:- Привет!

Б: - ЗАравствуй!

A:- Ну как ты?

Б: - Ой/ вообще не могу//

Как муж отреагирует в этой ситуачии?

A: -

Abbildung 3: Auf eine Aussage über das Befinden reagieren 


\section{Item 4}

Прочитайте Аиалог между матерью (М) и сыном (C). Что спрашивает мать?

$\mathrm{M}:-$

C: - Аа вроде нет// А чё нужно-то?

М: - Хиеба/ и корма Аля собаки.

C: $-\Lambda$ адно/ через час.

Abbildung 4: Den Sohn zum Einkaufen bewegen

\section{Item 5}

Вы с подругой в ресторане.

Вы: - Что будем брать?

Она: - Не знаю.

Вы [официанту]:

Abbildung 5: Den Kellner um die Speisekarte fragen

Welchem soziopragmatischen Konstrukt nach GeR die einzelnen Items entsprechen, ist aus Tab. 2 ersichtlich: Spalte 2 der Tabelle benennt inhaltliche Elemente aus entsprechenden Skalen des GeR. Diese Zuordnung zu einzelnen Niveaustufen orientiert sich zum einen an der sprachlichen Kommunikationssituation, die durch das Item vorgegeben ist; zum anderen hat das Konstrukt auch die Frage nach dem expected response (format) zu beantworten. D. h. erwartet wird ein limited production task, da die Antwort nur aus wenigen Worten bestehen soll (was sich in der Anleitung des Tasks durch die Angabe einer entsprechenden Wortanzahl zeigen müsste, die von den ProbandInnen nicht überschritten werden darf, um die reliable Vergleichbarkeit der Antworten zu erhöhen).

Qian \& Pan (2014: 860) weisen in diesem Zusammenhang auf zwei Funktionen des expected response hin: „[...] [R]esponse format is deemed to be one of the variables that not only will help reflect the test construct to a certain extent but also may produce undesirable intervening effects on the test taker's performance 
and impact the rating process." Sie betonen zudem die Notwendigkeit, den $e x-$ pected response bei der Erstellung von Testspezifikationen mitzudenken, was auch mit den Studierenden besprochen wurde, im Rahmen dieses Beitrages aber nicht näher ausgeführt werden kann. Wichtig erscheint jedoch eine Einordnung der Items in das bereits unter Tab. 1 angeführte Konstrukt, sodass sichergestellt ist, dass die Schwierigkeit der Items auch der zu testenden Niveaustufe B1 entspricht und die Erwartungen an die TestkandidatInnen in Bezug auf ihre soziopragmatische Kompetenz von ihnen auch erfüllt werden, selbst wenn die Möglichkeit besteht, dass TestkandidatInnen anders reagieren, als die TesterstellerInnen erwarten: „Testees may not approach the testing situation in the expected manner“ (ebd.: 863). Diese Überlegung war u. a. auch ein Grund, warum Studierende von der schriftlichen Umsetzung des Items 2 in Form einer SMS-Kommunikation abgewichen sind. TestkandidatInnen hätten nonverbal reagieren und die Aufgabe lösen können, indem sie bspw. ihrer Sprechintention mit einem der folgenden Emoticons $:-;$, :- oder $:$ Ausdruck verliehen hätten.

Tabelle 2: Soziopragmatisches Konstrukt der Testitems 1-5

\begin{tabular}{|c|c|c|c|c|c|c|}
\hline & & Item 1 & Item 2 & Item 3 & Item 4 & Item 5 \\
\hline & & B1 & B1 & A2 & B2 & A2 \\
\hline $\mathrm{C} 1$ & $\begin{array}{l}\text { Idiomatische } \\
\text { Redewendungen }\end{array}$ & & $\checkmark$ & & & \\
\hline $\mathrm{B} 2-\mathrm{C} 2$ & Kohärenz und Kohäsion & & & & $\checkmark$ & \\
\hline B2-C1 & $\begin{array}{l}\begin{array}{l}\text { Differenziertes } \\
\text { sprachliches Register }\end{array} \\
\end{array}$ & & & & & \\
\hline $\mathrm{B} 1-\mathrm{C} 2$ & Genauigkeit der Äußerung & & & & $\checkmark$ & \\
\hline $\mathrm{B} 1-\mathrm{C} 1$ & Flexibilität & & & & $\checkmark$ & $\checkmark$ \\
\hline B1 & $\begin{array}{l}\text { Sitten, Gebräuche } \\
\text { und Werte }\end{array}$ & & $\checkmark$ & & & \\
\hline
\end{tabular}




\begin{tabular}{|l|l|c|c|c|c|c|}
\hline A2-C1 & $\begin{array}{l}\text { Sprachliche Kennzeichnung } \\
\text { sozialer Beziehungen }\end{array}$ & $\checkmark$ & & $\checkmark$ & & \\
\hline A1-B2 & $\begin{array}{l}\text { Situationstypische } \\
\text { Redewendungen }\end{array}$ & $\checkmark$ & & & & $\checkmark$ \\
\hline \multirow{5}{*}{ A1-B1 } & $\begin{array}{l}\text { Sprachliche Höflichkeit } \\
\text { vs. sprachliche Grobheit }\end{array}$ & $\checkmark$ & & & & $\checkmark$ \\
\hline & $\begin{array}{l}\text { Neutrales sprachliches } \\
\text { Register }\end{array}$ & & & & & $\checkmark$ \\
\hline & $\begin{array}{l}\text { Lineares Verbinden und } \\
\text { Verknüpfen von Äußerungen }\end{array}$ & & & & $\checkmark$ & \\
\hline
\end{tabular}

Die später ermittelte Item-Schwierigkeit (zwischen 0,35 für Item 4 und 0,9 für Item 5) entspricht in etwa der hier vorgenommenen Zuordnung zu den einzelnen Kompetenzniveaus (grau markierte Zeile 2 in Tab. 2), die durch eine andere Studierendengruppe als jene der TesterstellerInnen erfolgte. Item 3 und Item 5 wurden einer niedrigeren Kompetenzstufe A2, Item 1 und Item 2 exakt der zu testenden Stufe B1 und Item 4 einer höheren Stufe, nämlich B2, zugeordnet. Der ermittelte Reliabilitätskoeffizient lässt auf eine einigermaßen konsistente $\mathrm{Zu}$ ordnung durch die Studierenden schließen $(\alpha=0,63)$. Weniger konsistent war die Gruppe hingegen, was ihre Einschätzung der Authentizität der Items im Vergleich mit real world items betraf $(\alpha=0,47)$.

\subsection{Ergebnisse 1}

Da eine Pilotierung der Testitems an einer Tiroler Schule mit Russischunterricht auf B1 nicht möglich war, wurde diese als Erstes im Rahmen des Seminars (SE) „Kommunikation - Text - Authentizität“ (LV3) mit einer Gruppe von Studierenden (Gruppe 1) simuliert, von denen die meisten Lernenden - abgesehen von jenen der HS und MS - ihre Sprachausbildung an der Universität begonnen hatten. Die 20 Studierenden des SE wurden durch Abzählen in fünf Untergruppen eingeteilt; es wurde anschließend darauf geachtet, dass in jeder dieser Untergruppen ein/e MS oder HS war. Innerhalb jeder Untergruppe löste 
jede/jeder einzelne Studierende die fünf Testitems, danach wurden die Ergebnisse einer anderen Untergruppe zugeteilt, um die „Richtigkeit“ der Antworten auf einer Skala von 0 bis 2 zu bestimmen. Unter „Richtigkeit“ war als Erstes die soziopragmatische Angemessenheit der Äußerung mit 2 (=angemessen), 1 (=wenig angemessen) oder 0 (=überhaupt nicht angemessen) festzustellen. Die sprachliche Korrektheit war dabei zweitrangig: Slips bzw. mistakes blieben gänzlich unberücksichtigt; errors brachten nur dann nicht die volle Punkteanzahl (2), wenn sie die Kommunikation empfindlich störten. ${ }^{8}$ Bei der Bewertung durch die Studierendengruppen wurde eine relativ hohe Übereinstimmung erreicht $(\alpha=0,75)$. Interessant, wenn auch wenig verwunderlich im Sinne des Tertiärspracheneffekts, ist die Tatsache, dass die soziopragmatische Angemessenheit bei der Lösung der Testitems umso höher war, je mehr Fremdsprachen der/die Studierende sprach.

Was die Schwierigkeit der Items hinsichtlich ihrer Lösung angeht, so ergab sich für Gruppe 1 ein Mittelwert von 6,7 (von 10 möglichen Punkten). Item 1 und 2 konnten von der Hälfte bzw. mehr als der Hälfte der Gruppe 1 gelöst werden; auch Item 3 und Item 5 mit einer Trennschärfe von 0,37 resp. 0,5 waren für viele Studierende relativ einfach (85\% bzw. 90\% hatten diese A2-Items gelöst); Item 4 nur 35\%. Die Zuordnung dieses Items zu B2, dessen Trennschärfe sehr niedrig ist $(0,02)$, legt nahe, dass es sich nicht in das Konstrukt der anderen Items eingliedert. Es wurde obendrein von den Studierenden als zu schwierig empfunden (50\% der Studierenden haben 0 Punkte erreicht), sodass vorgeschlagen wurde, es aus einem späteren Test zu entfernen (s. Tab. 3).

8 Ich bin mir der Tatsache bewusst, dass die Studierenden kein Bewertungstraining absolviert hatten und die Feststellung der Richtigkeit auf einer Skala von 0 (sprachlich nicht angemessen, inkorrekt) bis 2 (sprachlich angemessen, korrekt) bzw. deren Auswertung nur bedingt als reliabel anzusehen ist. Ziel dieser Bewertungsrunde war neben dem Feedback an die TesterstellerInnen v. a. eine Sensibilisierung der Lehramtsstudierenden für ihre zukünftige Rolle als BeurteilerIn im Beruf. 
Tabelle 3: Auswertung der Gruppe 1

\begin{tabular}{|c|c|c|c|c|c|c|}
\hline $\begin{array}{c}\text { Items } \\
1-5\end{array}$ & $\begin{array}{c}0 \\
\text { Punkte }\end{array}$ & $\begin{array}{c}1 \\
\text { Punkt }\end{array}$ & $\begin{array}{c}2 \\
\text { Punkte }\end{array}$ & $\begin{array}{c}\text { Item- } \\
\text { Mittelwert }\end{array}$ & $\begin{array}{l}\text { Mittelwert } \\
\text { gesamt }\end{array}$ & $\begin{array}{l}\text { Ranking nach } \\
\text { Schwierigkeit }\end{array}$ \\
\hline Item 1 & $15,0 \%$ & $35,0 \%$ & $50,0 \%$ & 1,35 & \multirow[t]{5}{*}{6,7} & Item 4 \\
\hline Item 2 & $5,0 \%$ & $30,0 \%$ & $65,0 \%$ & 1,60 & & Item 5 \\
\hline Item 3 & - & $15,0 \%$ & $85,0 \%$ & 1,85 & & Item 1 \\
\hline Item 4 & $50,0 \%$ & $15,0 \%$ & $35,0 \%$ & 0,85 & & Item 2 \\
\hline Item 5 & $35,0 \%$ & $25,0 \%$ & $40,0 \%$ & 1,05 & & Item 3 \\
\hline
\end{tabular}

Item 1 war aufgrund der eher ausführlichen und in schwieriger Sprache verfassten Anleitung dem Niveau B1 zugeordnet worden (s. Tab. 2). Dennoch wurden von den TeilnehmerInnen der Gruppe 1 viele sprachlich korrekte und höflich angemessene Reaktionen verfasst, die entweder eine Nullanrede (Извини) oder eine Begrüßung (Привет/Здравствуй) als Anrede enthielten, oder der fehlende Name wurde durch drei Punkte (...) ersetzt, in seltenen Fällen auch ergänzt (Пёmp/ Аимa). Gelegentlich wurden soziopragmatisch angemessene, aber grammatikalisch falsche Repliken geboten: Мой друг нет дома, могу я позвонить его? Neben der Personalform vy statt ty in der Anrede (Вы можете помогать мне?) kam es gelegentlich auch zu einer inkorrekten Wahl des Possessivpronomens: Извини, ть не можешь дать мне твой (statt: свой) телефон? In anderen Fällen traten stilistische Ungereimtheiten zutage: Скажи, пожалуйста, у тебя нет номер телебона Никалая? Я забыл клюи, а него нет дома. Блин! oder Ты не мог би мне помочь? Андрея нет дома. Можно мне воспользоваться твоим телефоном? Es gab aber auch sprachlich korrekte und soziopragmatisch angemessene Sprechakte: Привет! Можно позвонить с твоего телефона? Аай, пожалуйста, мобильник, [...].

Bei Item 2 (zugeordnet zu B1) fiel es den meisten zukünftigen RussischlehrerInnen in Gruppe 1 leicht, eine soziopragmatisch angemessene Antwort zu geben: Das Item war einem Lehrwerk entnommen und nur geringfügig verändert worden. Der direkte Austausch einer Emotion (Freude oder Überraschung) dürfte

9 Hier und im Folgenden werden alle Äußerungen genau so wie in der Testsituation wiedergegeben. Lediglich Unterstreichungen stammen vom Autor und dienen der Hervorhebung.

Anka Bergmann, Olga Caspers \& Wolfgang Stadler (Hg.)

Didaktik der slawischen Sprachen - Beiträge zum 1. Arbeitskreis in Berlin (12.-14.9.2016)

(C) 2018 innsbruck university press, ISBN 978-3-903187-11-5, DOI 10.15203/3187-11-5 
von den Studierenden - so wie der Austausch von Information - als vertraute Routineangelegenheit angesehen worden sein und wurde in den meisten Fällen zwar sprachlich einfach, aber durchaus adäquat und angemessen gelöst: $O$, каא хорошо! Я очень рад; Аа, уже знаюо! Аа, что твь! Он женится? bzw. Что? Не верю! Schwierigkeiten bei der Beurteilung zeigten sich - nicht nur bei diesem Item - immer wieder darin, ob Mündlichkeit (z. B. Intonation) in schriftlichen Texten überhaupt bewertet werden kann (Очень прекрасные новости.). Dennoch plädierten die Studierenden für eine schriftliche Ausführung der Beantwortung und gestanden ein, dass sie in einer mündlichen Testsituation wohl (noch) mehr Fehler gemacht hätten und dass sie dankbar waren, beim schriftlichen Verfassen der Lösung mehr Zeit zum Nachdenken gehabt zu haben, als dies in einem spontanen mündlichen Gespräch der Fall gewesen wäre (vgl. auch Nold \& Rossa 2008: 158).

Item 3 (A2) erweckte aufgrund seiner sprachlichen Einfachheit (äußerst vertraute Begrüßungs- und Routineformeln) den Eindruck, auch eine einfache Antwort generieren zu können. Sprachlich wiesen die meisten der Antworten auch kaum grammatikalische Unstimmigkeiten auf. Jedoch zeigte sich hier v. a. bei den NMS, dass sie auf die Wendung Oй/вообще не могy// [dt. in diesem Kontext vielleicht: Ojelich bin fix und fertig// bzw. Ojelich kann einfach nicht mehr//] soziopragmatisch auf der emotionalen Ebene wenig Adäquates erwidern konnten, offenbar, weil sie die Wendung nicht verstanden. Sie waren vielmehr der Meinung, nach dem Grund des Verhaltens fragen zu müssen oder sie vermuteten eine gesundheitliche Unpässlichkeit: Почемy?; Почему? Что с тобой?; Скажи, как был mвой денъ?; Тебе плохо?; Ты больна?

Die zu initiierende Replik in Item 4 - zugeordnet zu Niveaustufe B2 - stellte die meisten Studierenden mit Deutsch als Erstsprache vor große Schwierigkeiten. Bei keinem anderen Item blieben sie mehr Antworten schuldig als bei diesem. Das Problem lag z. T. im mangelnden Verstehen des Wortes вpode bzw. der Wendung Aa вpоде неm/l. Andere wiederum schätzten die Situation pragmatisch zwar richtig ein, konnten aber ihre Intention sprachlich nicht korrekt realisieren: Будешь в магазин? Можешь купить чуть-иуть?; Ты не можешь идти в магазин, пожалуйста?; Тъ скоро приедешь домой?

Item 5 war für die meisten Studierenden ein ,klares' A2-Item, einige ordneten es sogar der Stufe A1 zu. Probleme, die bei der Lösung auftraten, lagen zum einen

Anka Bergmann, Olga Caspers \& Wolfgang Stadler (Hg.)

Didaktik der slawischen Sprachen - Beiträge zum 1. Arbeitskreis in Berlin (12.-14.9.2016)

(C) 2018 innsbruck university press, ISBN 978-3-903187-11-5, DOI 10.15203/3187-11-5 
darin, dass der Dativ [официанту] des Adressaten nicht richtig gedeutet wurde und viele eine Replik des Kellners, statt an den Kellner, anboten: Я рекомендую сегодня дъгба с рисом и шардонн;; Вам надо помочь? Zum anderen wurde die Situation zwar richtig verstanden, aber sprachlich etwas unangemessen wiedergegeben, da nicht klar ist, wofür sich die SprecherIn bedankt: Mьг еще nодумаем. Сnасибо!

\subsection{Ergebnisse 2}

Dieselben fünf Items wurden zum Vergleich Gruppe 2, Lernenden an der Universität im dritten Semester, vorgelegt, die annähernd halb so lang wie Gruppe 1 Russisch studierten.

Tabelle 4: Auswertung der Gruppe 2

\begin{tabular}{|c|c|c|c|c|c|c|}
\hline $\begin{array}{c}\text { Items } \\
1-5\end{array}$ & $\begin{array}{c}\mathbf{0} \\
\text { Punkte }\end{array}$ & $\begin{array}{c}\mathbf{1} \\
\text { Punkt }\end{array}$ & $\begin{array}{c}\mathbf{2} \\
\text { Punkte }\end{array}$ & $\begin{array}{c}\text { Item- } \\
\text { Mittelwert }\end{array}$ & $\begin{array}{c}\text { Mittelwert } \\
\text { gesamt }\end{array}$ & $\begin{array}{c}\text { Ranking nach } \\
\text { Schwierigkeit }\end{array}$ \\
\hline Item 1 & $54,5 \%$ & $22,7 \%$ & $22,7 \%$ & 0,68 & \multirow{2}{*}{2,59} & Item 4 \\
\hline Item 2 & $27,3 \%$ & $54,5 \%$ & $18,2 \%$ & 0,91 & & Item 3 (5) \\
\hline Item 3 & $63,6 \%$ & $36,4 \%$ & - & 0,36 & & Item 5 (3) \\
\hline Item 4 & $72,7 \%$ & $27,3 \%$ & - & 0,27 & & Item 1 \\
\cline { 1 - 4 } Item 5 & $63,6 \%$ & $36,4 \%$ & - & 0,36 & & Item 2 \\
\hline
\end{tabular}

In Gruppe 2 (s. Tab. 4) wurden wesentlich niedrigere Mittelwerte $(M=2,59$; $\mathrm{SD}=1,29)$ als in Gruppe $1(\mathrm{M}=6,7 ; \mathrm{SD}=2,6)$ beobachtet. Dieser Unterschied konnte als signifikant nachgewiesen werden $(\mathrm{t}(40)=-6,578, \mathrm{p}<0,0001)$. Wählt man für einen faireren Vergleich aus Gruppe 1 jene Studierenden aus, die - so wie die meisten Studierenden in Gruppe 2 - Deutsch als Erstsprache haben, so ergibt sich für diese Untergruppe ein geringerer Mittelwert $(M=5,23 ; S D=1,96)$. Der Unterschied zu Gruppe 2 ist in diesem Fall nicht signifikant $(\mathrm{t}(31)=-1,738$, $\mathrm{p}=0,0921$ ).

Hinsichtlich soziopragmatischer Angemessenheit zeigt sich in Gruppe 2, dass Lernende häufig das Geschlecht des/der AdressatIn oder jenes/jener SprecherIn missachten, in dessen/deren Rolle sie schlüpfen sollten (могу я позвонить моему 
nодругу; я [сын] еще не знала). Zudem verwechseln sie - v. a. in Item 1 (Bitte an einen Freund) - die ty-lvy-Anrede (извините, меня возможнио позвонит с вашим телефоном моему другy) oder übernehmen in Item 5 - so wie auch einige TeilnehmerInnen aus Gruppe 1 - unverständlicherweise die Rolle des Kellners, anstatt den Kellner anzusprechen: Что вы хотели бъг? House (1993: 170), die ein Modell für die Analyse von inappropriate responses in der MS/NMS-Interaktion vorgelegt hat, spricht in diesem Fall von operational difficulties. Sehr oft wird aber das Schema nicht verstanden (conceptual deficiency, ebd.), die Interpretation der Kommunikationssituation durch die Studierenden ist eine andere, als es das übliche Skript nahelegt, das eine konventionalisierte und routinierte Handlungsabfolge darstellt. Diese Schwierigkeiten treten z. B. bei Item 3 (Аадно, другой раз.), Item 4 (Ты уже был в магазине?) und Item 5 (Скажите, пожалуйста, что ваша любимая блюда здесь?) auf.

Des Weiteren sind sprachliche Schwierigkeiten (language-based difficulties) zu erwähnen, wie falsche Imperative _Aadите нам еще пар минутьи, пожалуйста! (Item 3); falsche Kasusformen $У$ тебя есть времена? bzw. falsche Lexik $У$ нас нет вроде (Item 4) bzw. Минутку, пожслуйста. Мы уже не знаем (Item 5).

In einer abschließenden Analyse der Testitems stellte sich heraus, dass die Arbeitsanleitungen (rubrics) teilweise gekürzt, vereinheitlicht und die Sprache einem A2-Niveau angepasst werden müssten, um Verständnisschwierigkeiten auszuräumen, die das Lösen eines Items behindern. Bei Item 1 wurde die Angabe eines Vornamens für jenen Adressaten, der um das Handy gebeten wird, als nützlich für seine Anrede erachtet. Die Fragestellung Чmо Вы скажете / Как В ompeazupyeme? wurde zwar für den universitären Sprachunterricht, nicht aber für den Unterricht in der Schule als sinnvoll befunden und es wurde vorgeschlagen, diese auf Что тьг скажешь / Как ть отреагируешь? zu ändern. Wenig passend hinsichtlich AdressatInnengerechtheit wurde Item 3 erachtet, da dieser Dialog zwischen einem Ehepaar stattfindet. Es wurde vorgeschlagen, die GesprächspartnerInnen in Freund und Freundin zu ändern. Da sowohl Item 2 als auch Item 4 ein Gespräch zwischen Mutter und Sohn simulieren, wurde angedacht, eines der zwei Items auf ein Mutter-Tochter-Gespräch umzustellen. Die Beschreibung der einzelnen Kommunikationssituationen ist in Item 1 sehr ausführlich, in Item 5 äußerst minimalistisch dargestellt. Die Studierenden schlugen auch hier eine

Anka Bergmann, Olga Caspers \& Wolfgang Stadler (Hg.)

Didaktik der slawischen Sprachen - Beiträge zum 1. Arbeitskreis in Berlin (12.-14.9.2016)

(C) 2018 innsbruck university press, ISBN 978-3-903187-11-5, DOI 10.15203/3187-11-5 
Vereinheitlichung vor, die Länge der Rubriken und die Präzisierung der einzelnen Situationen betreffend. In Item 5 wurde der Gebrauch der Wendung $B 6 \imath c$ nодругой moniert und empfohlen, die Anleitung in Парень с девуикой в ресторане zu ändern, um Alter und Beziehung der GesprächspartnerInnen klar zu machen. Als Kritik wurde noch angeführt, dass drei der fünf Items reaktives Sprechen testen, nur zwei Items würden eine initiative Gesprächsführung überprüfen.

\subsection{Interpretation der Ergebnisse}

Die gegebenen Antworten zeigen exemplarisch, aber dennoch anschaulich, welche Charakteristika die russische Lernersprache auf unterschiedlichen Stadien aufweist und sie belegen die Schwierigkeiten beider Gruppen - von den MS und HS abgesehen -, die fünf Items aus soziopragmatischer Sicht angemessen lösen zu können.

Gruppe 1 war nicht nur in einem höheren Semester und somit auf einem sprachlich höheren Niveau, sondern aufgrund der Arbeit im Seminar auch anders für soziopragmatische Besonderheiten des Russischen sensibilisiert als Gruppe 2. Dieser Fakt erklärt die besseren Ergebnisse. Das Lernziel für Gruppe 2 (RII) lautet im Curriculum (2015) wie folgt: „Weitgehend selbstständige Sprachverwendung, einfacher Erfahrungs- und Informationsaustausch; Verstehen der Hauptpunkte mittelschwerer, mitunter auch längerer schriftlicher und mündlicher Texte" (Curriculum Bachelorstudium Lehramt Sekundarstufe 1.10.2016 ${ }^{10}$ ). Neben den vier Fertigkeiten Hören, Sprechen, Lesen und Schreiben sowie Lexik und Grammatik wird unter den Kursinhalten auch der „Ausbau der interkulturellen Kompetenz“ angeführt. Explizit wird - und das ist zu bedauern - weder die pragmatische noch die soziolinguistische Kompetenz in der Modulbeschreibung erwähnt. Auch wenn diese Kompetenzen nicht das vorrangige Ziel sind, so ist es dennoch verwunderlich, dass Gruppe 2 auf B1-Niveau derart geringe Leistungen im soziopragmatischen Bereich bietet. Item 4 war auch für diese 22 KandidatInnen das schwierigste Item, am leichtesten fielen Gruppe 2 die Items 1 und 2. Item 3 und 5 lagen von der Schwierigkeit dazwischen. Spezifisch fremdsprachliches

10 http://tinyurl.com/y9o69dku [02.12.2017].

Anka Bergmann, Olga Caspers \& Wolfgang Stadler (Hg.)

Didaktik der slawischen Sprachen - Beiträge zum 1. Arbeitskreis in Berlin (12.-14.9.2016)

(C) 2018 innsbruck university press, ISBN 978-3-903187-11-5, DOI 10.15203/3187-11-5 
Handlungswissen situationsadäquat, stilistisch angemessen und propositional genau einzusetzen, stellte für die meisten Lernenden eine große Schwierigkeit dar. Das „Wesentliche von dem, was man sagen möchte, verständlich auszudrücken" (GeR 2001: 129) wird von Gruppe 1 (ohne MS und HS) nur zur Hälfte $(M=5,23)$, von Gruppe 2 nur zu einem Viertel $(M=2,59)$ erreicht.

\section{Schlussfolgerungen}

Die Ergebnisse dieser Studie zeigen deutlich, dass auch im universitären Sprachunterricht soziopragmatische Kompetenz nur marginal entwickelt wird und keinesfalls an jene der MS und HS heranreicht. Dass in der Schule an diesen Kompetenzen noch weniger gearbeitet wird, scheint durch die Angaben im Fragebogen (s. Abschnitt 1) bestätigt: Nur 6,2\% der befragten Russischlehrkräfte geben an, während eines Schulhalbjahres soziopragmatische Kompetenz „oft“ zu bewerten (vgl. Drackert \& Stadler 2017). Die Studierenden schienen zwar die konstruierten Kommunikationssituationen größtenteils zu verstehen, sie waren aber nur in wenigen Fällen in der Lage, relevante und verständliche Antworten (relevant and comprehensible responses, vgl. House 1993: 163) zu geben. Die Arbeit mit Interjektionen und Partikeln sowie mit Routineformeln, die typisch für verschiedene Interaktionsschemata sind, sollte unbedingt in den Unterricht integriert werden, wie die Arbeit mit Studierenden in LV1 gezeigt hat. Ein weiterer Aspekt, an dem zu arbeiten wäre, sind direkte und indirekte Sprechakte bzw. Gesagtes vs. Gemeintes verstehen. Lernende nehmen vieles, was sie hören oder lesen, ,zu wörtlich', sie verstehen, was gesagt, nicht aber, was gemeint war bzw. verstehen nicht, warum etwas so gesagt wurde, wie es gesagt wurde. Nur so dürfte es zu erklären sein, dass jemand auf die Replik Oül вообще не могy// (Ojelich kann einfach nicht mehr/) mit Что ты не можешь? (Was kannst du nicht?) antwortet. Die Unangemessenheit dieser Äußerung „steht nicht im Einklang mit dem Diskurs und wird daher im Augenblick des Sprechens als nicht relevant erachtet", so erklärt House (ebd. 169) - mit Bezug auf Goofman - inappropriate responses. Vielleicht sind WDCT auch zu schwierig oder nicht wirklich geeignet, die soziopragmatische Kompetenz von Lernenden zu überprüfen, aber sie sind ein erstes und relativ einfach auszuwer-

Anka Bergmann, Olga Caspers \& Wolfgang Stadler (Hg.)

Didaktik der slawischen Sprachen - Beiträge zum 1. Arbeitskreis in Berlin (12.-14.9.2016)

(C) 2018 innsbruck university press, ISBN 978-3-903187-11-5, DOI 10.15203/3187-11-5 
tendes Format, bevor man sich in Untersuchungen anderen Testformaten, wie z. B. Elicited Imitation, R-DCT oder Rollenspielen zuwendet.

Leider gibt es noch immer viel zu wenig Untersuchungen mit ausreichendem Datenmaterial für die russische Lernersprache (s. Bergmann in diesem Sammelband), um die Gründe für (in)appropriate responses, wie sie in diesem Beitrag ebenfalls nur exemplarisch skizziert werden konnten, aufzuzeigen (vgl. House 1993: 161). Es wäre auch zu einfach, wenn dieser Beitrag eine Antwort auf die Frage geben könnte, wie man im (schulischen) Russischunterricht soziopragmatische Kompetenz entwickelt und bewertet. Nur wenn eine ausreichende Anzahl an Daten aus der russischen Lernersprache vorliegt, wird es möglich sein, auf die sprachlichen, konzeptuellen und operationalen Schwierigkeiten, die Lernleistungen hemmen, im Unterricht besser einzugehen. Manchmal übernehmen und imitieren Lernende soziopragmatische Kompetenzen aus ihrer Erstsprache; in vielen, wenn auch nicht in allen Situationen, sind sie damit erfolgreich. Und manchmal ist ihnen die Möglichkeit des Transfers gar nicht bewusst.

Um sicherzustellen, dass wirkungsvolle soziopragmatische Kompetenz auf B1-Niveau erreicht wird, wollen wir uns abschließend noch einmal das Konstrukt (Tab. 2) in Erinnerung rufen und für unsere Zwecke auf den Stufen A1B1 ergänzen (Tab. 5):

- Sprache und Register sind an einen neutralen Stil gebunden, die syntaktischen Verknüpfungen sind linear, sprachliche Höflichkeit ist an $t y / v y$ Formen und einfache Begrüßungsformeln (z. B. Привет vs. Здравствуйте) gekoppelt und situationstypische Redewendungen sind auf jene Situationen beschränkt, „denen man normalerweise (und auf Reisen) begegnet“ (GeR 2001: 33).

- Sprachliche Kennzeichnung (normaler) sozialer Beziehungen fallen u. a. in den Bereich Familie, FreundInnen, Schul- und ArbeitskollegInnen betreffen also die private und öffentliche Domäne (ebd.: 118).

- Sitten, Gebräuche und Werte der eigenen Kultur sollen mit jenen der Kultur der Zielsprache verglichen werden; „auf entsprechende Signale“ ist zu achten (ebd.: 122). 
- Regelbewusstsein und Flexibilität müssen in der Kommunikationssituation gegenübergestellt werden, um den SprachverwenderInnen zu demonstrieren, wie sie lernen können, routinemäßige Situationen zunehmend zu verlassen (ebd.: 131).

- Propositionale Genauigkeit ist selbst auf B1-Niveau noch begrenzt und Gedanken und Inhalte werden nicht immer so formuliert, „dass das Gemeinte klar wird“ (ebd.: 128).

Tabelle 5: Soziopragmatisches Konstrukt (A1-B1)

\begin{tabular}{|l|l|l|}
\hline \multirow{2}{*}{ Niveau } & \multicolumn{1}{|c|}{ Deskriptoren } & \multicolumn{1}{c|}{ Umsetzung } \\
\hline \multirow{2}{*}{ A1-B1 } & $\begin{array}{l}\text { Sprachliche Höflichkeit vs. } \\
\text { sprachliche Grobheit }\end{array}$ & $\begin{array}{l}\text { einfache, alltägliche Forme(l)n der } \\
\text { Begrüßung, der Anrede und des Befin- } \\
\text { dens etc.; positive - negative Höflichkeit }\end{array}$ \\
\cline { 2 - 3 } & Sprachliches Register & neutral \\
\cline { 2 - 3 } & $\begin{array}{l}\text { Verbinden und Verknüpfen } \\
\text { von Äußerungen }\end{array}$ & linear, zusammenhängend \\
\hline A1-B1 & $\begin{array}{l}\text { Situationstypische Redewen- } \\
\text { dungen }\end{array}$ & vertraut, normal \\
\hline A2 & $\begin{array}{l}\text { Sprachliche Kennzeichnung } \\
\text { sozialer Beziehungen }\end{array}$ & $\begin{array}{l}\text { normale Interaktion, Kontaktgespräche: } \\
\text { freundschaftlich, familiär, kooperativ }\end{array}$ \\
\hline B1 & $\begin{array}{l}\text { Sitten, Gebräuche und Werte } \\
\text { Konventionen der eigenen Kultur in } \\
\text { Gegenüberstellung zur zielsprachlichen } \\
\text { Kultur }\end{array}$ \\
\hline B1 & Flexibilität & $\begin{array}{l}\text { einfache sprachliche Mittel; sprachliche } \\
\text { Bewältigung des Alltagslebens }\end{array}$ \\
\hline B1 & Genauigkeit der Äußerung & $\begin{array}{l}\text { begrenzt bis hinreichend; verständliche } \\
\text { Kommunikation des Wesentlichen }\end{array}$ \\
\hline
\end{tabular}

Ein kommunikativer und handlungsorientierter RAF-Unterricht kann und darf die soziolinguistisch-pragmatischen Komponenten nicht ausklammern - das hat diese Studie eindeutig gezeigt. Nur so kann spezifisch fremdsprachliches Handlungswissen im gesteuerten Spracherwerb wirksam werden. Dennoch wird es für LehrerInnen nicht immer einfach sein zu klären, ob die Defizite, die inappropriate responses ausmachen und kennzeichnen, aus der mangelnden lexikalisch- 
grammatikalischen Sprachbeherrschung, dem mangelnden pragmatischen bzw. soziolinguistischen Wissen oder aus beiden Bereichen resultieren. Für ein konstruktives, formatives Feedback, das den Lernprozess positiv beeinflusst, wäre dieses Wissen aber eine essentielle Voraussetzung.

\section{Literaturverzeichnis}

Bachman, L. F. (1990). Fundamental Considerations in Language Testing. Oxford: Oxford University Press.

Bachman, L. F. \& Palmer, A. S. (2010). Language Assessment in Practice: Developing Language Assessments and Justifying Their Use in the Real World. Oxford, New York: Oxford University Press.

Blattner, G. \& Fiori, M. (2011). Virtual Social Network Communities: An Investigation of Language Learners' Development of Sociopragmatic Awareness and Multiliteracy Skills. CALICO Journal, 29 (1), 24-43. Verfügbar unter: https://tinyurl.com/ydeerdo6 [02.12.2017].

BMBWK (2004). Lehrplan AHS-Oberstufe Lebende Fremdsprachen. Verfügbar unter: http://tinyurl.com/yasf58dx [02.12.2017].

BMBWK (2006). Lehrplan AHS-Unterstufe Lebende Fremdsprachen. Verfügbar unter: http://tinyurl.com/yasf58dx [02.12.2017].

Curriculum Bachelorstudium Lehramt Sekundarstufe (Allgemeinbildung). Abschnitt 23: Unterrichtsfach Russisch (259-270). Verfügbar unter: https://tinyurl.com/y9o69dku [02.12.2017].

Drackert, A. \& Stadler, W. (2017). Leistungsbeurteilungskompetenz von Russischlehrerkräften in Deutschland, Österreich, der Schweiz und Südtirol (DACHS): Zwischen Status Quo und aktuellen Bedürfnissen. Zeitschrift für Fremdsprachenforschung, 28 (2), 233-258.

Ésmantova, T. (2011). Pjat' èlementov [uroven’ B1]. Sankt-Peterburg: Zlatoust.

GeR=Trim, J., North, B. \& Coste, D. (Hrsg.) (2001). Gemeinsamer europäischer Referenzrahmen für Sprachen: Lernen, lehren, beurteilen. Berlin: Langenscheidt.

Freie und Hansestadt Hamburg, Behörde für Schule und Berufsbildung (2009). Bildungplan gymnasiale Oberstufe. Neuere Fremdsprachen. Verfügbar unter: http://tinyurl.com/y7z522ev [02.12.2017].

House, J. (1993). Toward a Model for the Analysis of Inappropriate Responses in Native/Nonnative Interactions. In G. Kasper, Sh. Blum-Kulka (Hrsg.), Interlanguage Pragmatics (161-183). New York, Oxford: Oxford University Press. 
Kanik, M. (2013). Reverse Discourse Completion Task as an Assessment Test for Intercultural Competence. Studies in Second Language Learning and Teaching. (SSLLT), 3 (4), 621-644. Verfügbar unter: https://tinyurl.com/ydg56hac [02.12.2017].

Kasper, G. \& Roever, C. (2005). Pragmatics in Second Language Learning. In E. Hinkel (Hrsg.), Handbook of Research in Second Language Teaching and Learning (317-334). Mahwah, NJ: Lawrence Erlbaum Associates.

Kasper, G. \& Rose, K. R. (Hrsg.) (2001). Pragmatics in Language Teaching. Cambridge: Cambridge University Press.

Klieme, E. (Hrsg.) (2008). Unterricht und Kompetenzerwerb in Deutsch und Englisch. Ergebnisse der DESI-Studie. Weinheim, Basel: Beltz.

Kolotova, N. I. \& Kofanova, E. I. (2012). Formirovanie pragmatičeskoj kompetencii studentovfilologov v processe izučenija russkogo jazyka kak inostrannogo. Elektronnyj naučnyj žurnal Kurskogo gosudarstvennogo universiteta, 1 (21). Verfügbar unter: https://tinyurl.com/y7xxfp7p [02.12.2017].

Krulatz, A. (2014). Electronic Requests in Native and Non-Native Russian: Insights into Foreign Language Learners' Sociolinguistic Competence. Journal of Linguistics and Language Teaching, $5(1), 87-97$.

Krulatz, A. (2015). Judgments of Politeness in Russian: How Non-Native Requests Are Perceived by Native Speakers. Intercultural Communication Studies XXIV (1), 103-122. Verfügbar unter: https://tinyurl.com/y8omwj8u [02.12.2017].

Leech, G. N. (1983). Principles of Pragmatics. London: Longman.

Madyarov, I. (2014). Assessing Russian. In A. J. Kunnan (Hrsg.), The Companion to Language Assessment IV:19:138 (1. Aufl.) (2057-2067). John Wiley \& Sons.

Marasco, K., O’Rourke, C. et al. (2004). Pragmatic Language Assessment Guidelines. A Best Practice Document. ECICMC Standards and Guidelines Speech Sub-Committee. Verfügbar unter: https://tinyurl.com/ydf85rhq [02.12.2017].

Milejko, E. V. \& Stacenko, A. S. (2015). Formirovanie sociokul'turnoj kompetencii na zanjatijach po russkomu jazyku kak inostrannomu (na primere tekstov o Rossii). Filologičeskie nauki. Voprosy teorii i praktiki, 5 (47), 108-111. Verfügbar unter: https://tinyurl.com/y73onn3s [02.12.2017]. 
Mirzaei, A., Roohani, A. \& Esmaeili, M. (2012). Exploring Pragmalinguistic and Sociopragmatic Variability in Speech Act Production of L2 Learners and Native Speakers. The Journal of Teaching Language Skills (JTLS), 4 (3), 79-102. Verfügbar unter: https://tinyurl.com/y8h7zhmm [02.12.2017].

Nold, G. \& Rossa, H. (2008). Sprachbewusstheit Englisch. In E. Klieme (Hrsg.), Unterricht und Kompetenzerwerb in Deutsch und Englisch. Ergebnisse der DESI-Studie. Weinheim, Basel: Beltz.

Qian, D. \& Pan, M. (2014). Response Formats. In A. J. Kunnan (Hrsg.), The Companion to Language Assessment II:7:52 (1. Aufl.) (860-875). John Wiley \& Sons.

Riddiford, N. \& Joe, A. (2010). Tracking the Development of Sociopragmatic Skills. TESOL Quarterly, 44 (1), 195-205.

Roever, C. (2014). Assessing Pragmatics. In A. J. Kunnan (Hrsg.), The Companion to Language Assessment I:2:7 (1. Aufl.) (125-139). John Wiley \& Sons.

Stadler, W. (2015a). Teaching and Testing Sociopragmatics in the Russian Language Classroom. Athens Journal of Philology, 2 (3), 149-162. Verfügbar unter: https://tinyurl.com/ycc8pmfy [02.12.2017].

Stadler, W. (2015b). Prepodavanie i testirovanie sociopragmatičeskoj kompetencii RKI. In L.A. Verbickaja, K.A. Roganova, T.I. Popova et al. (Hrsg.), Russkij jazyk i literatura v prostranstve mirovoj kul'tury. Materialy XIII Kongressa MAPRJaL. Granada, Ispanija, 13-20 sentjabrja 2015 goda (363-369). Sankt-Peterburg: MAPRJaL.

Xiao, F. (2015). Proficiency Effect on L2 Pragmatic Competence. Studies in Second Language Learning and Teaching. (SSLLT), 5 (4), 557-581. Verfügbar unter: https://tinyurl.com/y7lltvzg [02.12.2017].

ŽRUG=Borisova, I. N., Vepreva, I. T., Ždanova, O. P. et al. (1995). Živaja reč' ural'skogo goroda: Teksty. Ekaterinburg: Izdatel'stvo Ural'skogo universiteta. 
